\title{
Editorial
}

\section{Ist die Zeitschrift für Soziologie eine Zeitschrift für Soziologie?}

\section{DOI 10.1515/zfsoz-2015-0999}

Die Benennung „Zeitschrift für Soziologie“ hat etwas Anmaßendes. Unterstellt sie doch eine Primärzuständigkeit, die faktisch so nicht gegeben ist. Andere soziologische Fachorgane sind da zurückhaltender; manche schließen in ihrem Titel ihren Entstehungsort oder weitere Disziplinen ein, andere verweisen eher offen auf einen Gegenstandsbereich. Auch wenn die Herausgeber der ZfS ihre Arbeit nicht im Sinne einer Primärzuständigkeit für die Disziplin verstehen - Zuständigkeit spielt eine wichtige Rolle. Eine Zeitschrift, die so heißt, sollte Beiträge versammeln, die - abgesehen von ihrer besonderen inhaltlichen Qualität - „Von innen“ zum soziologischen Binnendiskurs beitragen oder „von außen“, d.h. aus der Perspektive benachbarter Disziplinen, Themen behandeln, die für die Soziologie relevant sind. Bei der Auswahl von Manuskripten wird entsprechend darauf geachtet, dass ein Beitrag ein Erklärungsproblem soziologisch entwickelt und mit soziologischen Mitteln bearbeitet. Unabhängig von ihrer Qualität oder ihrem Anregungspotenzial werden beispielsweise Manuskripte mit einer dezidiert politik- oder wirtschaftswissenschaftlichen Ausrichtung abgelehnt oder es wird eine Überarbeitung mit soziologischer Akzentuierung empfohlen.

Das scheint zunächst ganz banal zu sein, denn es gibt immer Beiträge, die in fachlicher Hinsicht nicht so recht passen. Allerdings scheint es unserer Einschätzung nach zunehmend schwieriger zu werden, diese Zuordnung zu treffen. Bei vielen Manuskripten, die eigentlich soziologische Themen betreffen, ist es schwer, soziologische Bezüge auszumachen. Das betrifft zum Teil den methodischen, vor allem aber den konzeptionellen Zugriff. Sind Texte, die in ihrer Problemexposition weder auf soziologische Konzepte noch auf soziologische Theorietraditionen zurückgreifen, für eine Zeitschrift für Soziologie geeignet? Bemerkenswert ist, dass es sich nicht nur um Texte von Kolleginnen und Kollegen aus Nachbardisziplinen handelt, sondern auch um solche aus der Mitte der Disziplin. Geht man davon aus, dass eine Disziplin sich an einem eigenen Gegenstandsbereich, einer spezifischen Methodik und einer Theorietradition begründen kann, gibt es Anzeichen einer Entdisziplinierung auf allen drei Ebenen.
Die erste Entwicklung betrifft das wissenschaftliche Feld allgemein. So scheint es, dass (sozialwissenschaftliche) Forschung zunehmend über Gegenstände definiert wird, für die sich mehrere Disziplinen zuständig fühlen. Man betreibt Familien-, Migrations- oder Bildungsforschung, anstatt sich einen Gegenstand aus einer disziplinären Perspektive zu erschließen. Die Soziologie ist dabei unter „fremddisziplinären“ Druck geraten, seit sie weniger „die Gesellschaft“als Ganzes zu ihrem Gegenstand erklärt, sondern sich stärker dem Studium diverser Einzelphänomene sozialen Handels und sozialer Institutionen zugewendet hat. Dieser Druck hat sich erhöht, seit - etwa im Zuge des „ökonomischen Imperialismus“ - andere Disziplinen eine Ausweitung ihres Erklärungsanspruchs auf derartige Gegenstände reklamieren. Phänomene sozialer Kooperation und Normen werden heute anhand einheitlicher verhaltenswissenschaftlicher Forschungsdesigns transdisziplinär untersucht (Diekmann 2008). Auch unter dem neu entdeckten Etikett der „Kulturwissenschaften“ verwischen sich disziplinäre Grenzen an der Schnittstelle von Sprach-, Kunst-, Medien-, Geschichtswissenschaften, Ethnologie und Soziologie (Beer \& Koenig 2009).

Zweitens weichen bei der Methodenwahl klare disziplinäre Zuordnungen auf. Galt lange die Befragung als „Königsweg“ der Soziologie, der manch anderer Sozialwissenschaft fremd war, so wird heute auch in der Ökonomie und Psychologie verstärkt mit Befragungsdaten gearbeitet. Umgekehrt hat sich die soziologische Forschung gegenüber Experimenten und Verfahren der Text- und Bildinterpretation geöffnet. Empirische Studien lassen sich kaum mehr anhand einer spezifisch soziologischen Methodik beurteilen.

Die dritte, eher soziologieinterne Tendenz besteht darin, zunehmend auf ökonomische, psychologische oder kulturtheoretische Konzepte in der Erschließung eines Gegenstands zu rekurrieren anstatt auf genuin soziologische Forschungstraditionen. Gerade im Spektrum der Handlungstheorien haben sich zum ökonomischen Paradigma rationalen Handelns Varianten der sozialpsychologischen Einstellungsforschung (Theorie geplanten Verhaltens; Modell der Frame-Selektion) und der transdisziplinär inspirierten Kulturtheorien (Praxistheorie) gesellt. 


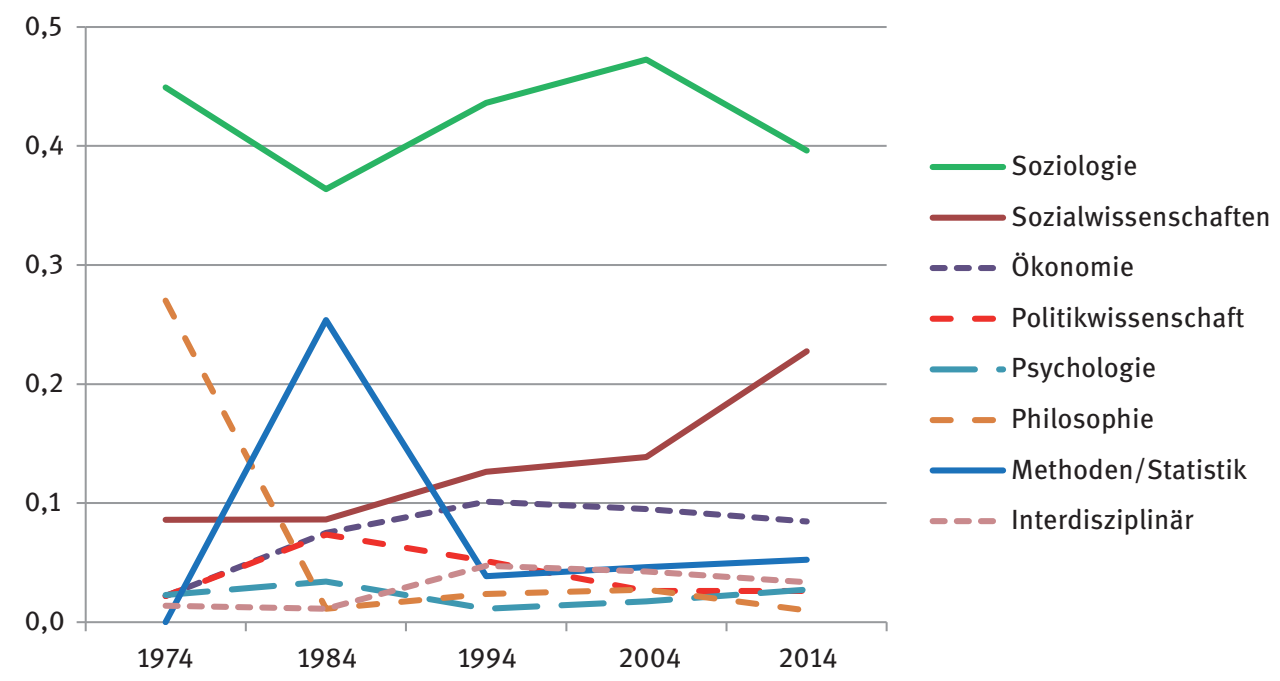

Abb. 1: Anteile der in ZfS-Beiträgen zitierten Literatur nach Disziplin, 1974-2014

Angesichts dieser mutmaßlichen, hier nur grob skizzierten Tendenzen stellt sich die Frage, welche Forschung sich eigentlich als „soziologische“ qualifizieren lässt. Und diese Frage ist für eine „Zeitschrift für Soziologie“ offensichtlich relevant.

Um die Bedeutung unterschiedlicher disziplinärer Einflüsse auf die soziologische Textproduktion weiter zu eruieren, haben wir eine kleine Untersuchung von Publikationen der ZfS durchgeführt. Über den Zeitraum der rund 40 Jahre des Bestehens der ZfS - sie erscheint seit 1972 - haben wir analysiert, welche disziplinären Bezüge die Bücher und Zeitschriftenartikel aufweisen, die in ZfS-Aufsätzen zitiert werden. Im Zitierverhalten manifestiert sich die Positionierung einer Publikation im wissenschaftlichen Feld besonders deutlich. Das Vorgehen erlaubt es allerdings nicht, die oben angesprochenen Dimensionen von Gegenstand, Methodik und Theorie gesondert abzugrenzen. Im Rahmen einer systematischen Zufallsauswahl haben wir jeweils die Hefte 1 und 3 der Jahrgänge 1974, 1984, 1994, 2004 und 2014 betrachtet und innerhalb dieser Hefte jeden der vier Beiträge untersucht. In jedem Beitrag wurden alle zitierten Quellen mit einer quantitativen Inhaltsanalyse kodiert. Im Fall von Fachzeitschriften wurde die Zuordnung anhand des Zeitschriftentitels, der disziplinären Selbstverortung der Zeitschrift auf ihrer Homepage und - bei Unklarheiten der disziplinären Ausrichtung der Artikel in der Zeitschrift vorgenommen. Im Fall von Büchern wurde anhand des Titels, Untertitels, Klappentextes und ggf. eines kursorischen Leseeindrucks über die Zuordnung entschieden. Sammelbandbeiträge wurden nach ihrem Thema, nicht nach der Ausrichtung des Sammelbandes kodiert.

Das Kategoriensystem unterscheidet das Spektrum etablierter Disziplinen. Als eigene Kategorie werden sozi- alwissenschaftliche Methoden und Statistik erfasst. Zwei separate Kategorien sind für Beiträge vorgesehen, die in disziplinübergreifenden Zeitschriften erschienen sind oder als Buch einen disziplinübergreifenden Charakter aufweisen. Zum einen werden dabei interdisziplinäre Referenzen innerhalb der Sozialwissenschaften erfasst (z. B. Fachzeitschriften wie Leviathan, Industrielle Beziehungen, Poetics oder Comparative Studies in Society and History; oder Bücher wie Esping-Andersens The Three Worlds of Welfare Capitalism). Zum anderen werden interdisziplinäre Publikationsorgane erfasst, die sozialwissenschaftliche Grenzen überschreiten oder komplett jenseits der Sozialwissenschaften zu verorten sind (z. B. Journal of Mind and Behavior, Fuzzy Sets and Systems oder Autonomous Agents and Multi-Agent Systems; oder Sammelbände wie Luhmann modelliert, herausgegeben von Thomas Kron). Berechnet wird nun, welcher Anteil den Referenzen einzelner Disziplinen an der Gesamtzahl der zitierten Quellen in einem ZfS-Beitrag zukommt. Die resultierenden Anteilswerte werden über die acht Beiträge eines Jahrgangs gemittelt. Die Anzahl von acht Aufsätzen ist als Minimalbasis für belastbare Aussagen anzusehen, denn je weniger Beiträge einbezogen werden, umso stärker reagieren die Durchschnittswerte auf die Ausrichtung einzelner Beiträge.

In den ZfS-Beiträgen werden durchschnittlich 33 (1974), 45 (1984), 84 (1994), 68 (2004) bzw. 81 (2014) Quellen zitiert. Kategorisiert man diese nach ihrer disziplinären Zugehörigkeit, ergeben sich die in Abbildung 1 dargestellten Anteilswerte. Demnach stammen je nach Jahrgang zwischen $36 \%$ (1984) und $47 \%$ (2004) der zitierten Quellen aus soziologischen Fachzeitschriften und Büchern. Die in der ZfS repräsentierte Forschung ist also schwerpunkt- 
mäßig in der soziologischen Literatur verankert, rezipiert aber in beträchtlichem Ausmaß Literatur über soziologische Grenzen hinweg. Und dies ist kein neues Phänomen, sondern es ist in fluktuierender Weise im gesamten Untersuchungszeitraum zu beobachten.

Betrachtet man die dabei hergestellten disziplinären Bezüge, werden sowohl Verschiebungen als auch Kontinuitäten sichtbar. Während 1974 gut jede vierte zitierte Quelle der Philosophie als damals zentraler Nachbardisziplin zuzuordnen war, sank dieser Anteil in den 1980er Jahren rapide und verblieb seither auf einem Niveau von etwa $2 \%$. Reflektiert wird darin der wissenschaftstheoretisch ausgerichtete Schulenstreit der Soziologie in den 1970er Jahren. Von den acht untersuchten ZfS-Publikationen des Jahres 1974 können sieben als Theoriebeiträge angesehen werden. Wie jüngst in einer anderen Inhaltsanalyse von ZfS- und KZfSS-Beiträgen gezeigt wurde, hat seitdem der Anteil empirischer Artikel immer mehr - auf heute rund $80 \%$ - zugenommen und die Relevanz der Philosophie zurückgedrängt (Kopp et al. 2012). Auch wenn sie keine ähnlich dominante Rolle wie die Philosophie in den 1970ern einnimmt, wird die Ökonomie ab den 1980er Jahren zur zentralen Bezugsdisziplin und erreicht einen stabilen Anteil von knapp unter 10\% der in der ZfS zitierten Literatur. Eine variierende Bedeutung mit 2-7 \% der zitierten Quellen hat Literatur aus der Politikwissenschaft. Die (Sozial-) Psychologie wird relativ stabil mit Zitationen in Höhe von 2-3\% bedacht. Gemessen daran, dass viele mikrosoziologische Positionen die Relevanz einer psychologischen Fundierung von Kognition und Handeln betonen, ist dies ein erstaunlich geringer Stellenwert. Während üblicherweise rund 5\% der Quellen Methoden- und Statistikliteratur sind, sticht der Ausreißer im Jahr 1984 ins Auge. Der ermittelte Anteil von $25 \%$ ist vermutlich eine stichprobenbedingte Überschätzung, die durch die geringe Anzahl von acht untersuchten ZfS-Beiträgen zustande kommt: Unter ihnen sind 1984 zwei Arbeiten der Methodenforschung sowie mehrere Beiträge der quantitativen Forschung. Außer den genannten Disziplinen überschreitet keine weitere Einzeldisziplin in einem der Jahrgänge die Marke von $5 \%$ der zitierten Literatur.

Unsere Wahrnehmung einer wachsenden Interdisziplinarität wird durch den kontinuierlichen Bedeutungszuwachs von Literatur belegt, die nicht genuin der Soziologie, sondern den Sozialwissenschaften insgesamt zuzuordnen ist. Von etwa 9\% im Jahr 1974 und 1984 steigt dieser Anteil auf rund 23\% im Jahr 2014. Dass die Interdisziplinarität gleichwohl Grenzen hat, zeigt sich am geringen Anteil interdisziplinärer Quellen, die die Sozialwissenschaften überschreiten und weit in den natur- oder geisteswissenschaftlichen Bereich hineinreichen. Seit 1994 schwankt dieser Anteil zwischen 3\% und 5\%.

Die Befunde können nicht ohne weiteres auf das Zitationsverhalten in der Soziologie verallgemeinert werden, da die ZfS wahrscheinlich eine selektive Auswahl an Autoren und Autorinnen anzieht bzw. in Folge des Peer ReviewVerfahrens eine selektive Auswahl von Manuskripten zur Publikation zulässt. Es ist somit denkbar, dass in anderen soziologischen Publikationsorganen eine andere Verteilung von Referenzdisziplinen anzutreffen ist.

Wie geht die ZfS mit den teils erkennbaren, teils nur vermuteten Tendenzen einer Entdisziplinierung um? Wir können uns solchen Entwicklungen sicher nicht verschließen und wollen nicht Gralshüter soziologischer Traditionen sein. Dennoch sollten Beiträge in der Zeitschrift für Soziologie ein hinreichendes Ausmaß an "Soziologie“ enthalten und diese Verortung mit Hilfe des behandelten Gegenstands, der eingesetzten Methoden und der theoretischen Bezüge herstellen.

\section{In eigener Sache}

Einen nun in der Tat exemplarisch soziologischen Beitrag möchten die Herausgeber an dieser Stelle herausheben. Marion Müllers Aufsatz über „The evils of racism and the wealth of diversity - Zum Bedeutungswandel bei den UNWeltkonferenzen gegen Rassismus“ wurde von der Fritz Thyssen Stiftung als bester sozialwissenschaftlicher Aufsatz des Jahrgangs 2014 mit dem ersten Preis ausgezeichnet. Die Zeitschrift für Soziologie gratuliert Marion Müller sehr herzlich. Die Herausgeber möchten sich aber auch bei der Fritz Thyssen Stiftung für diese Ehre herzlich bedanken.

Das Jahr 2015 ist für die Zeitschrift für Soziologie aber auch ein Jahr des Abschieds. Wir müssen Abschied nehmen von unserem langjährigen Hausverlag, dem Lucius \& Lucius Verlag. Wir danken Herrn Prof. Dr. Wulf D. von Lucius und seinen Mitarbeiterinnen und Mitarbeitern für eine stets vertrauensvolle, enge und freundschaftliche $\mathrm{Zu}$ sammenarbeit.

Einen außerordentlich großen Bruch bedeutet für die Herausgeber auch der Abschied von Hendrik Vollmer, der seit 2008 als Herausgeber und geschäftsführender Redakteur die Geschicke der Zeitschrift maßgeblich gelenkt hat. Das, was die Zeitschrift für Soziologie heute ist, verdankt sie ihm.

Die Herausgeber begrüßen unsere Konstanzer Kollegin Claudia Diehl in ihren Reihen. Sie bedanken sich an dieser Stelle bei Thomas Hinz außerordentlich für seine langjährige Herausgebertätigkeit und seine noch andau- 
ernde Unterstützung der Zeitschrift für Soziologie. Und schließlich freuen sich Redaktion und Herausgeber auf die Zusammenarbeit mit unserem neuen Hausverlag, dem VerlagDeGruyter Oldenbourg. Für die Zeitschrift für Soziologie sind mit diesem Wechsel viele Veränderungen verbunden, insbesondere ein neues Aussehen und ein neues Format. Zugleich bietet DeGruyter Oldenbourg der Zeitschrift für Soziologie und ihren Autorinnen und Autoren eine Reihe von neuen Publikations- und Darstellungsmöglichkeiten, über die wir demnächst informieren werden.

\section{Literatur}

Beer, B. \& Koenig, M. (2009): Grenzziehungen im System wissenschaftlicher Disziplinen - der Fall der „Kulturwissenschaft(en)“. Sociologia Internationalis 47: 3-38.

Diekmann, A. (2008): Soziologie und Ökonomie. Der Beitrag experimenteller Wirtschaftsforschung zur Sozialtheorie. Kölner Zeitschrift für Soziologie und Sozialpsychologie 60: 528-550.

Kopp, J., Schneider, J. \& Timmler, F. (2012): Zur Entwicklung soziologischer Forschung. Soziologie 41: 293-310.

Bielefeld, im Dezember 2015

Die Herausgeber 